\title{
Mitochondria in migraine pathophysiology - does epigenetics play a role?
}

\author{
Michał Fila ${ }^{1}$ Elżbieta Pawłowska², Janusz Blasiak ${ }^{3}$
}

${ }^{1}$ Department of Neurology, Polish Mother Memorial Hospital, Research Institute, Lodz, Poland

2Department of Orthodontics, Medical University of Lodz, Lodz, Poland

${ }^{3}$ Department of Molecular Genetics, University of Lodz, Lodz, Poland

Submitted: 12 February 2018

Accepted: 6 April 2018

Arch Med Sci 2019; 15 (4): 944-956

DOI: https://doi.org/10.5114/aoms.2019.86061

Copyright $\odot 2019$ Termedia \& Banach

\section{Abstract}

\author{
Corresponding author: \\ Michat Fila \\ Department \\ of Neurology \\ Polish Mother \\ Memorial Hospital \\ Research Institute \\ 188 Rzgowska St \\ 93-543 Lodz, Poland \\ Phone: +48 422711371 \\ Fax: +48 422711370 \\ E-mail michalfila@poczta. \\ onet.pl
}

The approximately three times higher rate of migraine prevalence in women than men may result from the mitochondrial transmission of this disease. Studies with imaging techniques suggest disturbances in mitochondrial metabolism in specific regions of the brain in migraine patients. Migraine shares some clinical features with several mitochondrial diseases and many other disorders include migraine headaches. Epigenetic regulation of mitochondrial DNA (mtDNA) is a matter of debate and there are some conflicting results, especially on mtDNA methylation. Micro RNAs (miRNAs) and long-noncoding RNA (IncRNAs) have been detected in mitochondria. The regulation of the miRNA-IncRNA axis can be important for mitochondrial physiology and its impairment can result in a disease phenotype. Further studies on the role of mitochondrial epigenetic modifications in migraine are needed, but they require new methods and approaches.

Key words: mitochondrial DNA, DNA methylation, micro RNA, long noncoding RNA.

\section{Introduction}

Migraine belongs to the commonest disorders worldwide and it significantly lowers quality of life and work productivity, resulting in a high burden for patients, society and employers. Annual migraine-associated cost in the US is about USD 80 billion [1].

Migraine prevalence varies from continent to continent and from country to country, but in general it is relatively high as compared with many other chronic diseases. That prevalence depends on age and can be approximately three times higher in women than in men (Figure 1). Hover, the peak of migraine incidence is observed in adolescence. It is a complex disease affecting several regions of the brain, inducing vascular dysfunction, cranial vasodilation and release of some neuromodulators, such as dopamine [2]. Current migraine therapies are effective only in a proportion of patients and studies on new therapeutic targets are needed.

Recurrent episodes of headache are the hallmark of migraine and they are usually associated with an array of other symptoms, including nausea, vomiting, photo- and phonophobia, visual disturbances and others. According to the International Headache Society, two main clinical subtypes of mi- 

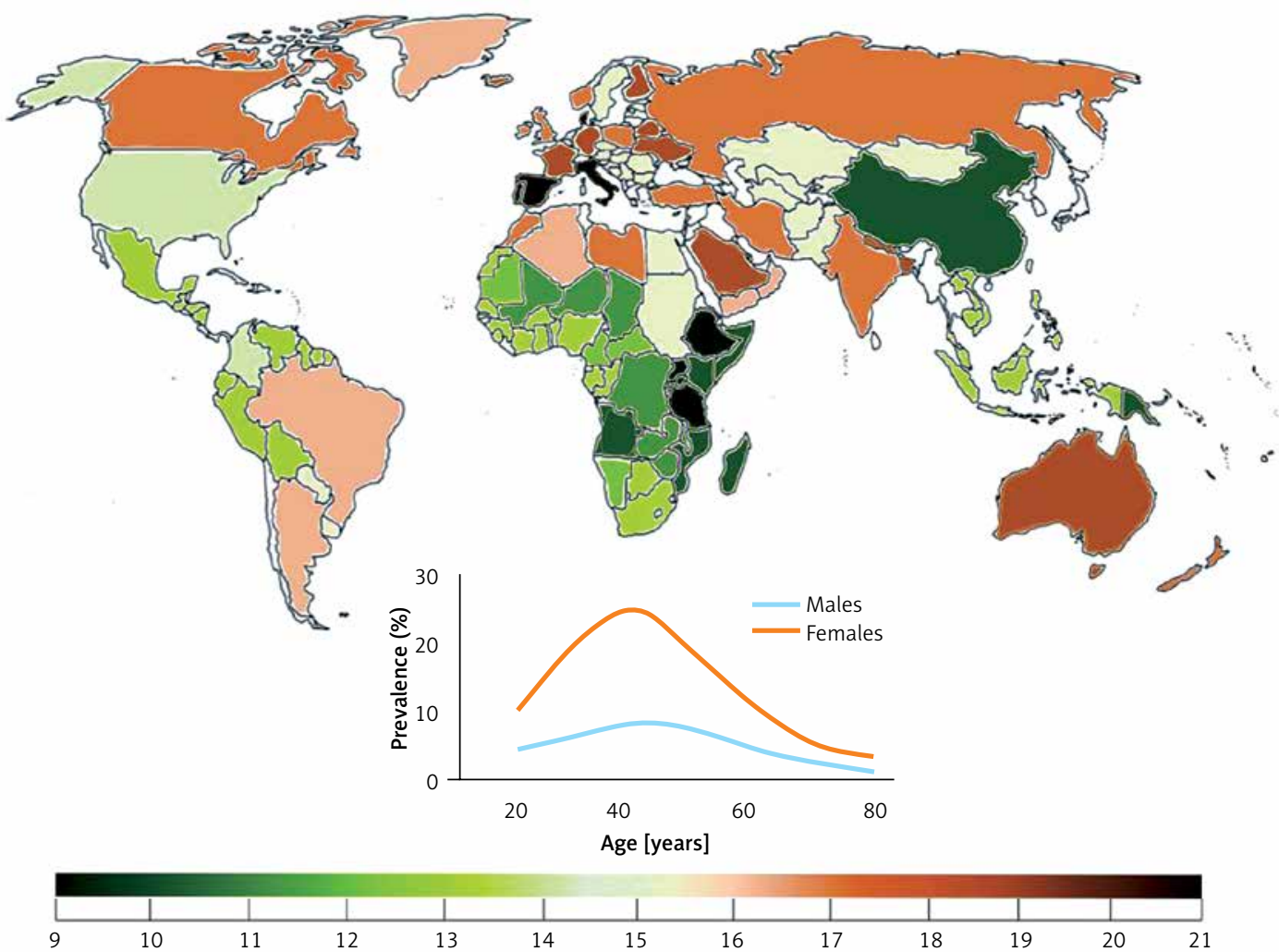

Figure 1. Worldwide migraine prevalence in 2016 according to WHO (both sexes, all ages). Inset - prevalence by age and sex (https://vizhub.healthdata.org/gbd-compare/)

graine can be considered: migraine with aura (MA) and without aura (MO). Other rarer type of migraine are basilar-type migraine (BTM) and familial hemiplegic migraine (FHM). Aura occurs in about one third of all migraine patients and includes mainly visual symptoms, but also aphasia, dyschromatopsia, prosopagnosia, ideational apraxia, alien hand syndrome and proper name anomia [3].

The pathophysiology of migraine is not fully known and there were two opposite theories on the cause and nature of migraine attacks - the vascular and the neuronal theory, which were then combined into the neurovascular theory [4]. Several other theories can be considered, including the hypothalamus theory, the serotonin theory, the depolarization theory and others. Migraine is a complex disease with the phenotype determined by the interaction between genetic, environmental and life-style factors. The pathogenesis of migraine is determined by phenomena occurring in the sensory cortex of the brain and/or the brain stem [5].

It is commonly accepted, although not firmly evidenced, that migraine is a threshold disease, released by a brain-related trigger [6-9]. Such migraine trigger and the wave of depolarization induce the activation of neurons of the trigeminal nerve, which release chemical substances inducing the dilation of blood vessels and inflammation, which directly cause migraine headache
(Figure 2). Cortical spreading depression (CSD), a wave of neuronal hyperexcitability followed by suppression of neural activity, may be responsible for disturbances occurring in MA [10].

Early magnetic resonance imaging (MRI) studies revealed a low level of high energy phosphates in the brain of migraine patients, suggesting defects in brain energy metabolism in migraine [11]. These studies had several limitations. First of all they did not provide information on physiological dysfunctions associated with observed effects. Currently, more detailed imaging studies on migraine mechanisms are conducted with positron emission tomography (PET) and functional MRI (fMRI) (see [12] for review). Studies with these techniques showed that repeated migraine attacks modified metabolism in specific regions of the brain involved in sensory and pain processing, including the bilateral insula, premotor and secondary somatosensory cortex (Figure 3). These studies reported ATP depletion due to a deficient mitochondrial metabolism in brains of migraine patients [13]. These studies suggest a significant role of mitochondria in the pathophysiology of migraine.

\section{Mitochondria - an important player in migraine pathogenesis}

Approximately three times higher frequency of migraine prevalence in females than males 


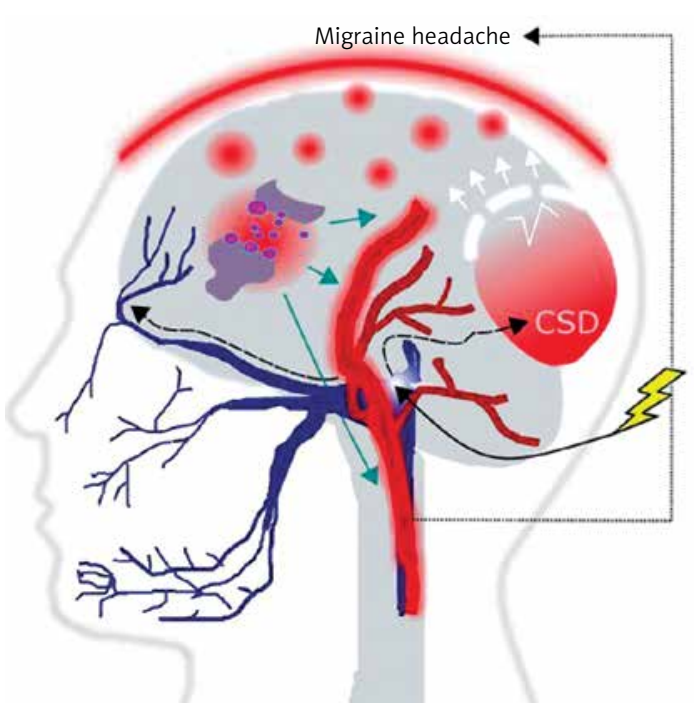

Figure 2. A migraine trigger (yellow thunder) acts on the nucleus (light blue oval) of the trigeminal nerve (dark blue) and activates it. Resulting waves of depolarization (black broken arrows) move along the nerve and reach the cortex, inducing cortical spreading depression (CSD). This results in an inflammation-like state and release of inflammatory neurotransmitters, which induce dilation of brain blood vessels (red), which in turn evokes migraine headache. This figure does not correspond to actual anatomical relationships within the brain

suggests either X-linked form of this disease, the mitochondrial transmission of another form of it, or both. However, if changes in mitochondria can contribute to the pathogenesis of migraine, not only mtDNA, but also the nuclear genes encoding mitochondrial components and regulating their functions may play a role.

Mitochondrial dysfunction can be associated with increased neuronal excitability resulting in increased susceptibility to migraine [14]. Any mitochondrial impairment in the brain likely leads to a deficit in mitochondrial energy, which in turn can be a migraine trigger. Some positive results on the influence of a high-fat and low-carbohydrate (ketogenic) diet on migraine and epilepsy course seem to support this assumption because such a diet stimulates mitochondrial metabolism [15, 16]. Further support is provided by the observation of the protective action of riboflavin and the Q10 coenzyme, both enhancing the function of the mitochondrial electron transport system (ETS, respiratory chain) against migraine [17-19].

A cross-sectional questionnaire-based study on 85 patients with various mitochondrial diseases (MDs) resulted in a higher frequency of headaches in this group compared to individuals without clinically detected mitochondrial impairments [20]. The highest prevalence of tension-type headache (TTH) was observed, but the prevalence of migraine and probable migraine was only slightly

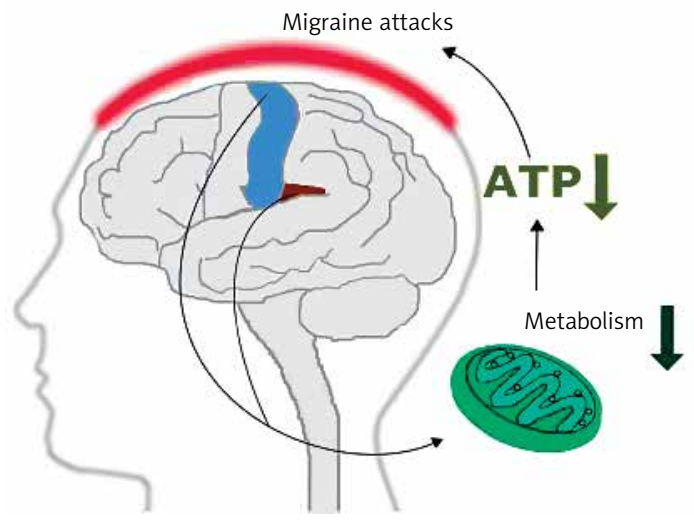

Figure 3. Repeated migraine attacks are associated with a metabolic modification of specific brain regions including premotor (blue) and secondary (brown) somatosensory cortex. Metabolic modifications in these regions result in impaired mitochondrial functions leading to depleted ATP production

lower. This study was supported by research showing a higher prevalence of migraine in individuals with MDs than in the general population [21]. Stratification of migraine cases into MD types suggested that migraine might not be a phenotypic feature of a particular MD, but might instead be linked with a common susceptibility of the central nervous system to some factors, likely associated with defects in ETS accompanying that MD.

\section{Riboflavin as well as MELAS and other mitochondrial diseases link migraine with mitochondria}

Riboflavin (vitamin $B_{2}$ ) belongs to the $B$ complex vitamins playing an important role as coenzymes in many flavoprotein enzyme reactions. It occurs in two active forms, flavin mononucleotide (FMN) and flavin adenine dinucleotide (FAD), which are important for vital mitochondrial processes, including metabolism of amino acids, purines and fatty acids as well as the redox reaction [22]. Riboflavin deficiency is a world-wide problem and it has been shown to improve the clinical picture of several mitochondrial diseases, including complex I and II-associated myopathies [23-29].

As brain metabolism is disturbed in migraine and riboflavin ameliorates the functioning of ETS, its evaluation in clinical trials as a prophylactic agent is justified [30]. Riboflavin prophylaxis was effective in migraine in adults, which may follow from the general neuroprotective effect of this compound [30-33]. Di Lorenzo et al. reported that migraine patients with non- $\mathrm{H}$ mLNA haplogroup responded more frequently to riboflavin than patients with the $\mathrm{H}$ group [34]. On the other hand, the frequency of riboflavin non-responders was higher in the $\mathrm{H}$ group. There were no differ- 
ences between MA and MO patients in riboflavin responsiveness. These studies suggest that the efficacy of riboflavin therapy can depend on the patient genotype.

Riboflavin decreased the frequency of headache attacks and their duration in a randomized, controlled trial performed by Shoenen et al. [35]. These results were confirmed in another clinical trial performed by Rahimdel et al., who also observed a decrease in frequency of headache attacks [36]. Several other clinical trials with riboflavin singly or in combination with other agents both in adult and adolescent migraine patients brought positive results - more details are presented in an excellent review by Thompson and Saluja [30].

Riboflavin was also reported to ameliorate mitochondrial dysfunction and protect against oxidative stress [22]. This compound protected against hyperglycemia and DNA damage in diabetic mice [37]. As we mentioned, the action of riboflavin may depend on the genotype of the recipient. Therefore, it is not very surprising that in some mitochondrial diseases which are associated with mutations, the efficacy of this drug can be low. However, riboflavin-based therapy is considered as effective or possibly effective at various levels of recommendation by several institutions, including the American Academy of Neurology and the American Headache Society (http://www. aan.com/guidelines).

Mitochondrial myopathy, encephalopathy, lactic acidosis, and stroke-like episodes (MELAS) is a complex, progressive neurodegenerative disorder with a great variability in clinical picture causing difficulty with distinguishing it from vascular cerebral diseases, first of all cerebral infarctions [38]. Migraine shares some clinical characteristics with MELAS, including headaches with nausea and vomiting [21]. Another mitochondrial disease, myoclonic epilepsy with ragged-red fibers (MERRF), is also associated with episodes of hemicranial headaches. This provokes the question whether migraine can be a monosymptomatic form of MELAS or MERRF. However, no association of mutations typical for MELAS with migraine was reported. Klopstock et al. did not observe any association between MA and mutations (deletions and substitutions) common in MELAS and MERRF [39]. In a subsequent study, Buzzi et al. did not observe any association between the m.3243A>G substitution in the tRNA ${ }^{\text {Leu }}$ gene and pure matrilineal multigenerational MA or MO [40]. In addition, neither patient enrolled in that study carried this mutation. No association of the m.3243A>G mutation with $\mathrm{MO}$ was observed [41]. In another study no association between nine point mutations (substitutions) and migraine with prolonged

Table I. Some clinical conditions associated with migraine [43]

\begin{tabular}{|c|c|}
\hline Condition & Disease \\
\hline \multirow[t]{5}{*}{ Genetic syndromes } & $\begin{array}{l}\text { Mitochondrial myopathy, encephalopathy, lactic acidosis, and stroke-like episodes } \\
\text { (MELAS) }\end{array}$ \\
\hline & $\begin{array}{c}\text { Cerebral autosomal dominant arteriopathy with subcortical infarcts and } \\
\text { leukoencephalopathy (CADASIL) }\end{array}$ \\
\hline & $\begin{array}{c}\text { Cerebral autosomal recessive arteriopathy with subcortical infarcts and } \\
\text { leukoencephalopathy (CARASIL) }\end{array}$ \\
\hline & Hereditary endotheliopathy with retinopathy, nephropathy, and stroke (HERNS) \\
\hline & Fabry disease \\
\hline \multirow[t]{2}{*}{ Vascular acquired diseases } & Strokes: ischemic and hemorrhagic episodes \\
\hline & Transient ischemic attack (TIA) episodes \\
\hline \multirow[t]{7}{*}{ Mitochondrial diseases } & Chronic progressive external ophthalmoplegia (CPEO) \\
\hline & Myoclonic epilepsy with ragged red fibers (MERRF) \\
\hline & Mitochondrial encephalomyopathy, lactic acidosis and stroke-like episodes (MELAS) \\
\hline & Mitochondrial neurogastrointestinal encephalomyopathy (MNGIE) \\
\hline & Myoclonic epilepsy with ragged-red fibers (MERRF) \\
\hline & Kearns-Sayre syndrome \\
\hline & Leber hereditary optic neuropathy \\
\hline \multirow[t]{2}{*}{ Other diseases } & Pediatric cyclic vomiting syndrome \\
\hline & Epilepsy \\
\hline
\end{tabular}


aura was observed [42]. Therefore, variability of mtDNA typical for MELAS is not reported to occur in migraine, and so these results do not support the thesis on migraine as a monosymptomatic form of MELAS. However, these studies, although carefully designed, were performed on relatively small populations. Table I presents some diseases associated with migraine [43].

Energy deficiency is especially important in muscles and neural tissue, particularly when combined with other stress, and contributes to many symptoms of mitochondrial myopathies [44]. Such myopathies associated with neurological disturbances are called mitochondrial encephalomyopathies. Several neurological symptoms occur also in migraine, including headaches, hearing impairment and seizures [45]. Therefore, some mitochondrial encephalomyopathies and migraine can share similar pathways of pathogenesis and so it is reasonable to look for a genetic background of migraine in mtDNA [46]. Moreover, this association allows one to speculate on the connection between migraine and epigenetic changes, which are reported for migraine comorbid disorders $[47,48]$.

\section{The human mitochondrial genome and its changes related to migraine}

The human mitochondrial genome is a closed, double-stranded DNA (mtDNA) molecule of $16,569 \mathrm{bp}$, containing 37 genes encoding 2 rRNAs, 22 tRNAs and 13 polypeptides (Figure 4). All polypeptides encoded by mtDNA are subunits of ETS: 7 subunits of complex I (NADH: ubiquinone oxidoreductase), 1 subunit of complex III (ubiquinol:cytochrome c oxidoreductase), 3 subunits of com- plex IV (cytochrome c oxidase) and 2 subunits of complex V (ATP synthase). The entire complex III and all remaining subunits of complexes I, II, IV and $V-79$ subunits in total - are encoded by nuclear DNA. Two strands of mtDNA are called heavy $(\mathrm{H})$ and light $(\mathrm{L})$ due to their base composition. mtDNA, in contrast to its nuclear counterpart, is densely packed with genetic information - more than 90 percent of it contains coding sequences, genes are intron-less and the ATPase 6 and ATPase 8 genes overlap. A small non-coding mtDNA fragment is called the control region, as it contains almost all control elements of mtDNA metabolism. Due to the high variability of human mtDNA its variants are classified into haplotypes [49].

ETS is built from mitochondrial- and nuclearencoded components and provides energy for the entire cell. However, even normally functioning ETS produces reactive oxygen species (ROS) and its malfunctioning increases ROS level. The importance and function of mitochondria should not be limited to energy production as this organelle plays an important role in calcium homeostasis and thermogenesis, the intrinsic pathway of apoptosis, signal transduction and other phenomena [50].

In humans, mtDNA does not recombine and is perpetuated exclusively in the maternal lineage. Therefore, it is useful for tracking genetic alterations through many generations. However, there are many mtDNA copies in a single cell, and not all of them need to be mutated to give a disease phenotype [51]. Therefore, mtDNA could also be exploited to track epigenetic changes sent from one generation to the next. However, epigenetic

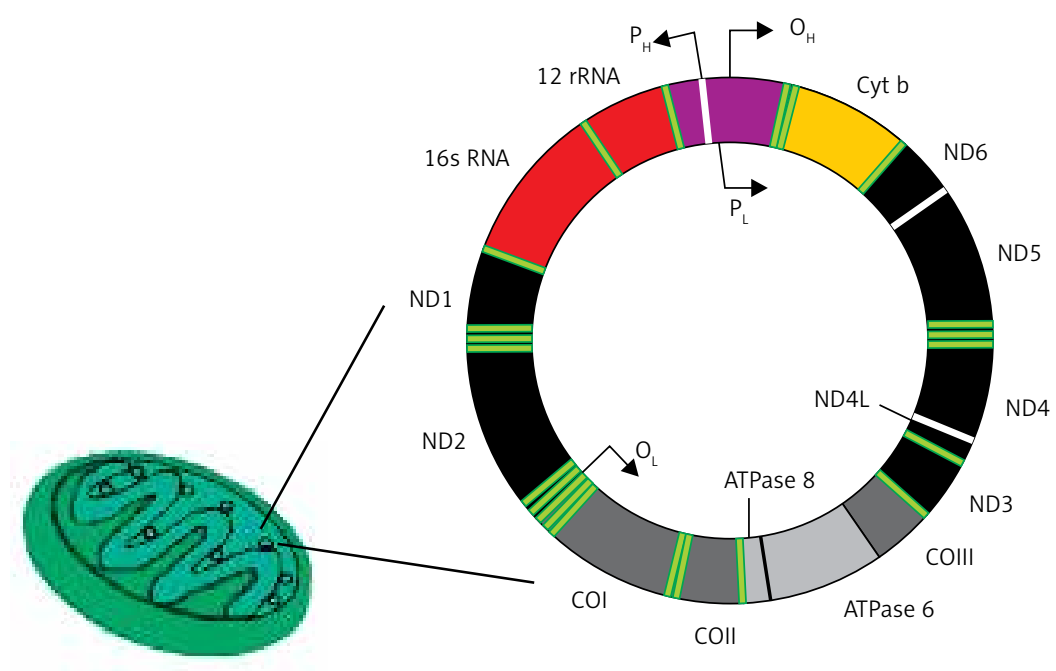

Figure 4. Human mitochondrium is a double membrane-bound organelle found in the cytoplasm. It usually contains several copies of mtDNA, which is a closed double-stranded DNA having heavy $(\mathrm{H})$ and light $(\mathrm{L})$ strands. It has 13 polypeptide encoding genes - Cytb, ND1-6, ND4l, ATPase 6 and 8, COI-III - whose products are components of the mitochondrial electron transport chain. Other genes of mtDNA are 2 rRNA and 22 tRNA genes. Promoters (P) for these genes ( 2 for heavy strand and 1 for light strand) are located in a single regulatory region, where also the origin for heavy strand replication occurs $\left(\mathrm{O}_{\mathrm{H}}\right)$ 
modifications to mtDNA have been far less exploited and are less well known than those to nuclear DNA.

mtDNA is characterized by greater sequence variation than its nuclear counterpart, but a cell usually has many mitochondria, which can have different genotypes. Therefore, wildtype mtDNA can occur along with its mutated variants in a single cell. This state is termed heteroplasmy in contrast to homoplasmy, which corresponds to the same sequence of a mtDNA locus in all mitochondria. Therefore, when a disease-associated variant occurs in a heteroplasmic form, its phenotypic consequences can be difficult to predict. In an extreme case, a lethal mutation in some mitochondria can be counterbalanced by the normal variant in remaining organelles. This creates a complex relationship between genetic variants in mtDNA and diseases. At a cellular level, a point mutation is considered to be expressed phenotypically with the threshold $80-90 \%$, but for a larger deletion the threshold is lower - 50-60\% [52]. At the tissue level, the proportion of mutants can positively correlate with the severity of the disease [53].

As mentioned, a strong connection between migraine and mitochondria was evidenced by the observation that migraine-affected individuals have impaired brain energy metabolism [54]. Mitochondria play a vital role in energy production, apoptosis regulation and production of ROS even in normal conditions [55]. These ROS are important in cell signaling, but their excess can lead to damage to cellular molecules [56]. The main source of ROS is oxidative phosphorylation (OXPHOS), producing ATP from ADP and inorganic phosphate $\left(P_{i}\right)$, powered by the transfer of electrons from NADH/FADH $H_{2}$ to $\mathrm{O}_{2}$ [57]. This transfer is executed by mitochondrial ETS containing four large multiprotein complexes, designated I-IV, located in the inner mitochondrial membrane, each having several electron carriers. The fifth complex, ATP synthase, accepts protons transported from the inner mitochondrial membrane, which fuel ATP productions. Additionally, inner membrane-bound ubiquinone and cytochrome $c$, located in the intermembrane space, play an important role in OXPHOS.

Oxidative stress is considered as a factor in migraine pathogenesis and is associated with an excess of ROS, which can damage DNA. However, Geyik et al. found no difference in oxidative stress markers, total oxidant status (TAS) and oxidative stress index (OSI) between migraine patients and controls [58]. An increased level of 8-hydroxy-2'-deoxyguanosine (8-oxoG) in plasma of migraine patients was observed in that study and this increase was more pronounced in $\mathrm{MO}$ than MA patients. 8-oxoG is a product of oxidative DNA damage, which may lead to mutation if not repaired or misrepaired, and it is considered as an oxidative stress marker in humans. In similar studies, Alp et al. observed that serum TAS levels were lower in patients with $\mathrm{MO}$ as compared to controls and ROS levels in serum were higher in these patients [59]. Patients displayed higher values of OSI than controls, who, in turn, exhibited a higher levels of total SH groups, which were negatively correlated with headache duration. On the other hand, OSI was positively correlated with headache frequency. These studies have several limitations, including the small number of patients enrolled less than 100 migraine cases in either study. Moreover, the studies were performed in a non-target tissue and the parameters for assessing oxidative stress were chosen rather arbitrarily and they do not decide about the occurrence and intensity of oxidative stress. Furthermore, a higher level of 8-oxoG can result from impairment in the DNA repair system or in general an impaired DNA damage response. 8-oxoG is mainly removed by the base excision repair (BER) system and in humans the hOGG1 glycosylase cuts the $N$-glycosylic bond between 8-oxoG and the DNA sugar $[60,61]$. However, the results presented in those papers are a weak rationale to causatively correlate migraine with oxidative stress and allocate the source of this stress to mitochondria.

The brain is highly dependent on oxidative metabolism of glucose and therefore it is sensitive to changes in the functioning of mitochondria, which is supported by the association of several neurodegenerative diseases with mitochondrial dysfunction [62-64].

Peroxisome proliferator-activated receptor- $\gamma$ coactivator-1 $\alpha$ (PGC-1 $\alpha)$ is an important regulator of the expression of genes involved in mitochondrial biogenesis and cellular reaction to oxidative stress $[65,66]$. Altered levels of mRNA of the PGC-1 $\alpha$ gene along with a decreased number of mtDNA copies were observed in trigeminal neurons in a rat model of migraine [67].

\section{Variability of mitochondrial DNA in migraine}

The mitochondrial genome has limited autonomy as thousands of proteins which can be found in mitochondria are encoded in the nucleus. Therefore, the variability of nuclear genes involved in mitochondrial functioning may be important in migraine pathogenesis. The problem of genetic variability in migraine is connected with migraine hereditability. An identical twin study resulted in the conclusion of approximately 50\% migraine hereditability [68].

A positive association between the m.4336A>G mutation, located on the $t R N A^{G l n}$, gene and sensorineural hearing loss and/or migraine was observed [69]. Wang et al. found an association between 
the occurrence of childhood cyclic vomiting, often considered as a migraine-like disease, and two rare heteroplasmic sequence variations containing 6 point and 2 length variants located in the mtDNA control region [70]. Two single nucleotide polymorphisms, m.16129G >A and m.16176C >T, in that region were associated with childhood cyclic vomiting. Moreover, these authors found that the homoplasmic variants located in the nt 1604016188 segment of the highly variable region 1 (HV1) important in the regulation of mtDNA replication were associated with both cyclic vomiting syndrome and migraine without aura. In another study, Zaki et al. found an association between two mtDNA polymorphisms, m.16519C $>T$ and m.3010G >A, and migraine headache and cyclic vomiting syndrome [71]. A higher, about three times, prevalence of migraine in individuals with the m.3243A>G mutation was reported [72]. This prevalence was observed in both $\mathrm{MO}$ and MA as well as in men and women separately. No association between the m.11084A $>\mathrm{G}$ polymorphism and migraine occurrence, whether MA or MO, in a Japanese population was found [73]. No difference between occurrence of the m.11084A $>G$ mutation in MA and MO patients was observed in a Danish population [74]. This mutation was detected neither in migraine patients, MA or MO, nor controls. It has functional significance as it replaces adenine with guanine in the gene encoding the ND4 subunit of ETS complex I, resulting in the substitution of threonine with alanine. Interestingly, this mutation was reported to occur in a substantial fraction (about $25 \%$ ) of Japanese migraineurs and was absent in tension-type headache patients and individuals with no migraine [75].

Mitochondrial haplogroup $U$, represented by the $12308 \mathrm{~A}>\mathrm{G}$ polymorphism, can play a role in migraine pathophysiology as this polymorphism was reported to associate with an increased risk of occipital stroke [76, 77]. Also other haplogroups in mtDNA can be directly or indirectly related to migraine. Marcuello et al. reported an association between the J haplogroup and efficacy of energy production by ETS [78]. As mentioned, migraine is associated with energy deficit, so that haplogroup and its polymorphisms of mtDNA may influence the susceptibility to migraine as well as to other diseases associated with energy deficit.

Generally, there are few studies on the association between mtDNA variants and migraine occurrence and these studies are usually performed on relatively small populations. Therefore, they should be continued on larger cohorts. Moreover, studies on variants of nuclear genes encoding mitochondrial components and influencing mitochondrial function should be further developed, especially as they are free from several difficulties in mtDNA research, including heteroplasmy requiring sequencing of the entire mitochondrial genome with a high coverage. Therefore, further studies on the role of mtDNA disturbances in migraine are justified and needed.

\section{Epigenetics of mtDNA and its potential in migraine}

Epigenetics deals with mechanisms of the regulation of gene expression, which are not directly associated with changes in DNA sequences. Three major epigenetic mechanisms are related to DNA methylation, histone modifications and RNA regulation.

Two aspects of epigenetic regulation in migraine pathophysiology can be considered. One is related to transient changes in epigenetic profile, which may result in changes in phenotype. The other is associated with such changes in the epigenetic pattern, which are perpetuated from one generation to the next and contribute to familial cases of the disease.

Epigenetic therapy, i.e. therapy targeting the epigenetic profile of specific genes, is considered in many human disorders, first of all cancer, in which several epigenetic modifiers are tested in phase I and II clinical trials [79]. Such therapy is also considered in migraine [80]. Valproate, a histone deacetylate inhibitor, has been successfully tested in prophylaxis of several classes of migraine [81-85]. However, its action cannot be unequivocally associated with an epigenetic mechanism as valproate is an anticonvulsive drug with several modes of action [86-88]. Moreover, it is not known how valproate acts on mitochondria.

Passaro et al. found a direct correlation between epigenetic markers of the $\mathrm{H} 3$ histone associated with genes involved in neuronal plasticity and CSD in rat brain [86]. This study suggests a potentially important role of epigenetic modifications in migraine pathogenesis, which was supported by subsequent studies [89-91]. Moreover, this importance has been recently confirmed by Gerring et al., who have identified 62 independent regions in DNA isolated from peripheral blood of migraine patients, which were specifically methylated [89].

Many migraine comorbid mitochondrial diseases are associated with changes in the epigenetic profile [46]. Therefore, it is justified to explore the role of epigenetic modifications to mtDNA in migraine pathogenesis. However, the issue of epigenetics in mitochondria is still a matter of debate, with some controversial aspects [92]. As mtDNA is not associated with histones, its epigenetic modifications are limited in comparison with its nuclear counterpart, but mtDNA occurs in many copies and each of them could have a different epigenetic profile. Therefore, DNA methylation and regulatory RNAs are major players in epigene- 
tic control of gene expression in mitochondria. In humans, DNA methylation of the CpG islands located in the promoters of many genes is of a particular significance. It is induced by DNA methyltransferases (DNMTs) $1 / 2$ and 3A/B [93, 94]. However, it should be stressed that mtDNA does not contain CpG islands, such as those targeted by DNA methyltransferases in the nucleus, but dispersed CPG dinucleotides occur in the mitochondrial genome [95]. Mitochondrial DNA methyltransferase 1 (mtDNMT1), an isoform of nuclear DNMT1, plays an important role in regulation of the expression of mitochondrial genes by modification of the mtDNA methylation profile [88]. This enzyme was reported to bind mtDNA in the region of the D-loop containing all three promoters needed for the transcription of mitochondrial genes, suggesting that mtDNMT1 can regulate gene expression. It was shown that DNMT1 interacted with CpG dinucleotides within mtDNA [96]. There is another DNA methyltransferase in the nucleus, DNMT3L, which, on binding with unmethylated lysines in $\mathrm{H} 3$ histone, guides DNMT3A/3B to DNA [97]. As there is no $\mathrm{H} 3$ histone in mitochondria, the potential of this enzyme seems to be lower than remaining DNA methyltransferases. However, DNMT3L is involved in DNA methylation in the nucleus, so it can play a role in the expression of genes whose products are important for mitochondrial homeostasis.

Another DNA methyltransferase, DNMT3A, was localized in the mitochondria of neurons and its decreased activity was reported to be associated with an abnormal mtDNA methylation pattern in amyotrophic lateral sclerosis, a progressive neurological disease [98]. However, it should be taken into account that in contrast to mtDNMT1, DNMT3A was not specifically targeted to mitochondria, but was found in these organelles. The identification of DNA methyltransferases operating in mitochondria shed light on the epigenetic mechanisms in these organelles, but on the other hand, it has added to the complexity of this issue [99]. Other epigenetics-related proteins detected in mitochondria are DNMT3B and two proteins involved in active demethylation, ten-eleven translocation (TET) 1 and 2 [100-103]. These methyltransferases are not only encoded in the nucleus, but are isoforms of nuclear proteins. Therefore, precise regulation of their expression and functions is required for cellular homeostasis and disease-related changes in their activity can result from an imbalanced nuclear-mitochondrial cross-talk [104]. However, some reports question the process of mtDNA cytosine methylation, indicating limitations of techniques employed in earlier studies [105].

The third aspect of epigenetics, RNA regulation, is first of all based on three kinds of non-coding (nc) regulatory RNAs: short ncRNA, including micro RNA (miRNA) of 22-23 bases in length (b) and piwi-interacting RNA (piRNA) of 26-31 b; medium ncRNA of 50-200 b and long non-coding RNA (IncRNA) longer than $200 \mathrm{~b}$ [106].

Micro RNA regulation is important in the nervous system [107, 108]. RNA interference components, including miRNAs and Argonaute proteins, were localized to mitochondria, showing another aspect of the cross-talk between the nucleus and mitochondria through an array of miRNAs, which are called "mitomiRs" [108].

Long non-coding RNAs are another element of epigenetic regulation, and in the nuclear genome it is associated with several important aspects of the regulation of gene expression, including chromosome $X$ inactivation [109]. IncRNA regulation is important in many cancers, including brain tumors [110, 111].

The mitochondrial transcriptome is quite complex and should not be limited to $37 \mathrm{mtDNA}$ genes, as almost the whole mitochondrial genome is transcribed $-99.9 \%$ of $\mathrm{H}$ and $97.6 \%$ of the $\mathrm{L}$ strand were reported to undergo transcription [112]. Many antisense transcripts can be found within the mitochondrial transcriptome. Moreover, many RNAs encoded in the nucleus are present in mitochondria and vice versa - several mitochondria-borne RNAs were reported to operate in the nucleus, indicating the importance of the mitochondria-nucleus cross-talk [113]. Altogether, many both "short" and "long" regulatory RNAs, including miRNAs and IncRNAs, can be found in mitochondria, and they can play an important role in the regulation of gene expression. In general, two species, miRNA and IncRNA, seem to be mutually regulated within the miRNA-IncRNA axis giving the functional balance between IncRNAs and miRNAs - the IncRNA-miRNA paradigm [114]. Departures from this paradigm can contribute to disturbances in energy production and can affect other processes in mitochondria important for the cell fate, which may result in disorders [115]. However, to our knowledge the role of IncRNA, either nuclear or mitochondrial, in migraine pathophysiology has not been explored so far. An association between disturbances in IncRNA functioning and mitochondrial impairment was observed in Down syndrome [116].

The mitochondria-nucleus cross-talk can also be expressed by the changes in the nuclear epigenetic profile reflected in mitochondria functioning, which in turn results in the global changes to the whole organism, as in the case of mitochondrial lifespan extension dependent on stress [117].

Several loci associated with migraine in the human genome have been identified in genome-wide association studies (GWAS) [118, 119]. Some of them can be linked with epigenetic mechanisms 
[120]. However, it should be underlined that no genes directly involved in establishing and maintaining the epigenetic profile, such as DNA methyltransferases or histone deacetylases, have been found in these studies. Moreover, GWAS studies did not provide results on the link between mtDNA variability and migraine.

\section{Conclusions and perspectives}

Epigenetic mechanisms are an important element of the control of gene expression. Transcriptional regulation through RNA molecules is essential for many genes $[121,122]$. On the other hand, the epigenetic profile undergoes changes induced by environmental conditions. Oxidative stress is linked with pathophysiology of many diseases, including migraine. However, in most cases it is not completely known whether the stress belongs to the reasons or consequences of a disease, or both. It is difficult to find a disorder not linked to oxidative stress in its pathogenesis. On the other hand, ROS are normally produced in mitochondria. Therefore, many oxidative stress-related disorders can be potentially linked with mitochondria. Consequently, association of a mitochondrial dysfunction with a clinically detectable disorder may result from a false positive correlation. Oxidative stress, resulting from impaired functioning of ETS, may lead to the accumulation of damage to cellular components and mitochondrial aging, cellular senescence and premature organismal aging. So far, no mtDNA damage in migraine has been reported, but the extent of mtDNA damage in the brain of mammals was negatively correlated with maximal life span [22].

Abnormal expression of PGC- $1 \alpha$ in neurons of a rat model of migraine provokes a question about the potential role of this protein in the pathogenesis of human migraine. Moreover, the involvement of PGC- $1 \alpha$ in mitochondrial biogenesis prompts the search for the role of mitophagy, a key element in the regulation of mitochondrial number, in migraine pathogenesis [123].

Migraine can be diagnosed as an isolated headache with autonomic symptoms or as a part of a syndrome associated with other complex diseases, including mitochondrial disorders. There is no general agreement whether these two categories of migraine are different or not. Some reports suggest that there are essential differences between these two forms of migraine not resulting from individual susceptibility to this disease. These differences are claimed to manifest first of all with a prolonged aura and stroke-like episodes in migraine associated with other diseases. However, Vollono et al. recently observed migraine without aura in about $80 \%$ of mitochondrial disease cases [43]. Therefore, it may be difficult to distinguish autonomous migraine from migraine associated with other diseases.

Several mitochondrial encephalomyopathies share neurological symptoms with migraine, which may follow from a common mitochondrial element in their pathogeneses. As no migraine-specific mutations in mtDNA have been identified yet, it is likely that this common element can be of epigenetic nature. The brain needs energy in the form of ATP, produced mainly in mitochondria, and disturbance in its supply can result in an increased sensitivity to migraine attack.

Complex mitochondrial diseases cannot be related exclusively to mtDNA as they are associated with mitochondrial proteins encoded by nuclear genes. Therefore, a complex genomic analysis including both mitochondrial and nuclear components of the human genome should be performed in research on pathogenesis of these diseases. Mitochondrial variation need to be studied in large family pedigree studies to find a correlation between mtDNA mutation and migraine to eventually identify true causal variants. Moreover, these studies should also include variability in relevant nuclear genes. Currently, the MitoProteome $\mathrm{Hu}$ man Mitochondrial Protein Database lists 3,625 proteins, including isoforms and splice variants, which can be allocated to mitochondria (http:// mitoproteome.org/).

Riboflavin proved to be effective in migraine prophylaxis in several clinical trials. However, some issues concerning the methodology of these trials as well as basic questions on riboflavin pharmacokinetics and pharmacogenomics should be addressed. These concern first of all interpretation of results of trials with $n$ placebo groups and determination of optimal doses of riboflavin as the mechanism of its absorption is not completely known.

In clinical practice, it is important to distinguish migraine from monosymptomatic forms of other diseases manifested by headaches, first of all MELAS, especially considering that migraine can share morbidity with some mitochondrial disorders.

Mitochondria add complexity to migraine pathogenesis. Therefore, new integrated approaches are needed to better understand migraine pathophysiology and translate molecular research into clinical practice to improve diagnosis and therapy of this disease.

The question from the title of this paper remains open. First of all, we must extend and deepen our knowledge on epigenetic regulation in mitochondria. mtDNA has a specific structure, which can constitute a barrier in the determination of mtDNA epigenetic pattern with methods established for its nuclear counterpart, as was shown for cytosine methylation in the D-loop. Therefore, the initial enthusiasm associated with mtDNA 
methylation should be revised [118]. This is especially important as the functional relevance of mtDNA methylation has been supported only by association studies and no reliable mechanistic research on this subject has been performed. Secondly, although accumulating body of evidence suggests the involvement of mitochondria in migraine pathogenesis, the exact mechanism of this involvement is unknown. The potential role of mitochondrially enriched miRNAs (mitomiRNAs) in migraine pathogenesis should be investigated.

GWAS provided information on many loci which can be linked to migraine, so epigenome-wide association studies with several high-throughput analyses may bring more information on the role of epigenetics in migraine. However, the evaluation of the significance of mitochondrial genome/ epigenome in migraine pathophysiology requires new methods and approaches.

\section{Conflict of interest}

The authors declare no conflict of interest.

\section{References}

1. Gooch CL, Pracht E, Borenstein AR. The burden of neurological disease in the United States: a summary report and call to action. Ann Neurol 2017; 81: 479-84.

2. Charles A. The pathophysiology of migraine: implications for clinical management. Lancet Neurol 2017; 17: 174-82.

3. Vincent MB, Hadjikhani N. Migraine aura and related phenomena: beyond scotomata and scintillations. Cephalalgia 2007; 27: 1368-77.

4. Warfvinge K, Edvinsson L. Pearls and pitfalls in neural CGRP immunohistochemistry. Cephalalgia 2013; 33: 593-603.

5. Ho TW, Edvinsson L, Goadsby PJ. CGRP and its receptors provide new insights into migraine pathophysiology. Nature Rev Neurol 2010; 6: 573-82.

6. Aurora SK, Welch KM, Al-Sayed F. The threshold for phosphenes is lower in migraine. Cephalalgia 2003; 23: 258-63.

7. Mulleners WM, Chronicle EP, Palmer JE, Koehler PJ, Vredeveld JW. Visual cortex excitability in migraine with and without aura. Headache 2001; 41: 565-72.

8. Reyngoudt H, Paemeleire K, Descamps B, De Deene Y, Achten E. 31P-MRS demonstrates a reduction in highenergy phosphates in the occipital lobe of migraine without aura patients. Cephalalgia 2011; 31: 1243-53.

9. Young W, Shaw J, Bloom M, Gebeline-Myers C. Correlation of increase in phosphene threshold with reduction of migraine frequency: observation of levetiracetam-treated subjects. Headache 2008; 48: 1490-8.

10. Chen SP, Ayata C. Spreading depression in primary and secondary headache disorders. Curr Pain Headache Rep 2016; 20: 44.

11. Montagna P. Magnetic resonance spectroscopy in migraine. Cephalalgia 1995; 15: 323-7.

12. Russo A, Silvestro M, Tedeschi G, Tessitore A. Physiopathology of migraine: what have we learned from functional imaging? Curr Neurol Neurosci Rep 2017; 17: 95.
13. Sarchielli P, Tarducci R, Presciutti O, et al. Functional $1 \mathrm{H}-\mathrm{MRS}$ findings in migraine patients with and without aura assessed interictally. Neuroimage 2005; 24: 1025-31.

14. Sparaco M, Feleppa M, Lipton RB, Rapoport AM, Bigal ME. Mitochondrial dysfunction and migraine: evidence and hypotheses. Cephalalgia 2006; 26: 361-72.

15. Di Lorenzo C, Coppola G, Sirianni G, et al. Migraine improvement during short lasting ketogenesis: a proofof-concept study. Eur J Neurol 2015; 22: 170-7.

16. Neal EG, Chaffe H, Schwartz RH, et al. A randomized trial of classical and medium-chain triglyceride ketogenic diets in the treatment of childhood epilepsy. Epilepsia 2009; 50: 1109-17.

17. D’Onofrio F, Raimo S, Spitaleri D, Casucci G, Bussone G. Usefulness of nutraceuticals in migraine prophylaxis. Neurol Sci 2017; 38: S117-120.

18. Gaul C, Diener HC, Danesch U. Improvement of migraine symptoms with a proprietary supplement containing riboflavin, magnesium and Q10: a randomized, placebo-controlled, double-blind, multicenter trial. J Headache Pain 2015; 16: 516.

19. Slater SK, Nelson TD, Kabbouche MA, et al. A randomized, double-blinded, placebo-controlled, crossover, addon study of CoEnzyme Q10 in the prevention of pediatric and adolescent migraine. Cephalalgia 2011; 31: 897-905.

20. Kraya T, Deschauer M, Joshi PR, Zierz S, Gaul C. Prevalence of headache in patients with mitochondrial disease: a cross-sectional study. Headache 2018; 58: 45-52.

21. Vollono C, Primiano G, Della Marca G, Losurdo A, Servidei S. Migraine in mitochondrial disorders: prevalence and characteristics. Cephalalgia 2018; 38: 1093-106.

22. Barja G, Herrero A. Oxidative damage to mitochondrial DNA is inversely related to maximum life span in the heart and brain of mammals. FASEB J 2000; 14: 312-8.

23. Kennedy DO. B Vitamins and the brain: mechanisms, dose and efficacy - a review. Nutrients 2016; 8: 68.

24. Bernsen PL, Gabreels FJ, Ruitenbeek W, Hamburger HL. Treatment of complex I deficiency with riboflavin. J Neurol Sci 1993; 118: 181-7.

25. Darin N, Hedberg-Oldfors C, Kroksmark AK, Moslemi AR, Kollberg G, Oldfors A. Benign mitochondrial myopathy with exercise intolerance in a large multigeneration family due to a homoplasmic m.3250T>C mutation in MTTL1. Eur J Neurol 2017; 24: 587-93.

26. Henriques BJ, Lucas TG, Gomes CM. Therapeutic approaches using riboflavin in mitochondrial energy metabolism disorders. Curr Drug Targets 2016; 17: 1527-34.

27. Liu XY, Wang ZQ, Wang DN, Lin MT, Wang N. A historical cohort study on the efficacy of glucocorticoids and riboflavin among patients with late-onset multiple acyl-CoA dehydrogenase deficiency. Chin Med J (Engl) 2016; 129: 142-6.

28. Olsen RKJ, Konarikova E, Giancaspero TA, et al. Riboflavin-responsive and -non-responsive mutations in $F A D$ synthase cause multiple Acyl-CoA dehydrogenase and combined respiratory chain deficiency. Am J Hum Genet 2016; 98: 1130-45.

29. Thakur K, Tomar SK, Singh AK, Mandal S, Arora S. Riboflavin and health: a review of recent human research. Crit Rev Food Sci Nutr 2017; 57: 3650-60.

30. Thompson DF, Saluja HS. Prophylaxis of migraine headaches with riboflavin: a systematic review. J Clin Pharm Ther 2017; 42: 394-403. 
31. Daniel O, Mauskop A. Nutraceuticals in acute and prophylactic treatment of migraine. Curr Treat Options Neurol 2016; 18: 14

32. Marashly ET, Bohlega SA. Riboflavin has neuroprotective potential: focus on parkinson's disease and migraine. Front Neurol 2017; 8: 333.

33. Namazi N, Heshmati J, Tarighat-Esfanjani A. Supplementation with riboflavin (Vitamin B2) for migraine prophylaxis in adults and children: a review. Int J Vitam Nutr Res 2015; 85: 79-87.

34. Di Lorenzo C, Pierelli F, Coppola G, et al. Mitochondrial DNA haplogroups influence the therapeutic response to riboflavin in migraineurs. Neurology 2009; 72: 1588-94.

35. Schoenen J, Jacquy J, Lenaerts M. Effectiveness of highdose riboflavin in migraine prophylaxis. A randomized controlled trial. Neurology 1998; 50: 466-70.

36. Rahimdel A, Zeinali A, Yazdian-Anari P, Hajizadeh R, Arefnia E. Effectiveness of vitamin B2 versus sodium valproate in migraine prophylaxis: a randomized clinical trial. Electron Physician 2015; 7: 1344-8.

37. Alam MM, Iqbal S, Naseem I. Ameliorative effect of riboflavin on hyperglycemia, oxidative stress and DNA damage in type-2 diabetic mice: mechanistic and therapeutic strategies. Arch Biochem Biophys 2015; 584 10-9.

38. Malhotra K, Liebeskind DS. Imaging of MELAS. Curr Pain Headache Rep 2016; 20: 54.

39. Klopstock T, May A, Seibel P, Papagiannuli E, Diener HC, Reichmann $H$. Mitochondrial DNA in migraine with aura. Neurology 1996; 46: 1735-8.

40. Buzzi MG, Di Gennaro G, D’Onofrio M, et al. mtDNA A3243G MELAS mutation is not associated with multigenerational female migraine. Neurology 2000; 54: 1005-7.

41. Di Gennaro G, Buzzi MG, Ciccarelli O, et al. Assessing the relative incidence of mitochondrial DNA A3243G in migraine without aura with maternal inheritance. Headache 2000; 40: 568-71.

42. Rozen TD, Shanske S, Otaegui D, et al. Study of mitochondrial DNA mutations in patients with migraine with prolonged aura. Headache 2004; 44: 674-7.

43. Vollono C, Primiano G, Della Marca G, Losurdo A, Servidei S. Migraine in mitochondrial disorders: prevalence and characteristics. Cephalalgia 2018; 38: 1093-106.

44. DiMauro S, Bonilla E, Zeviani M, Nakagawa M, DeVivo DC. Mitochondrial myopathies. Ann Neurol 1985; 17: 521-38.

45. Chomyn A, Enriquez JA, Micol V, Fernandez-Silva P, Attardi G. The mitochondrial myopathy, encephalopathy, lactic acidosis, and stroke-like episode syndrome-associated human mitochondrial tRNALeu(UUR) mutation causes aminoacylation deficiency and concomitant reduced association of mRNA with ribosomes. J Biol Chem 2000; 275: 19198-209.

46. Roos-Araujo D, Stuart S, Lea RA, Haupt LM, Griffiths LR. Epigenetics and migraine; complex mitochondrial interactions contributing to disease susceptibility. Gene 2014; 543: 1-7.

47. Qureshi IA, Mehler MF. Epigenetic mechanisms underlying human epileptic disorders and the process of epileptogenesis. Neurobiol Dis 2010; 39: 53-60.

48. Sun H, Kennedy PJ, Nestler EJ. Epigenetics of the depressed brain: role of histone acetylation and methylation. Neuropsychopharmacology 2013; 38: 124-37.

49. Morgan-Richards M, Bulgarella M, Sivyer L, et al. Explaining large mitochondrial sequence differences within a population sample. R Soc Open Sci 2017; 4: 170730.

50. Papa S, Martino PL, Capitanio G, et al. The oxidative phosphorylation system in mammalian mitochondria. Adv Exp Med Biol 2012; 942: 3-37.

51. Moraes CT, Schon EA, DiMauro S, Miranda AF. Heteroplasmy of mitochondrial genomes in clonal cultures from patients with Kearns-Sayre syndrome. Biochem Biophys Res Commun 1989; 160: 765-71.

52. Rossignol R, Faustin B, Rocher C, Malgat M, Mazat JP, Letellier T. Mitochondrial threshold effects. Biochem J 2003; 370: 751-62.

53. Chinnery PF, Hudson G. Mitochondrial genetics. Br Med Bull 2013; 106: 135-59.

54. Uncini A, Lodi R, Di Muzio A, et al. Abnormal brain and muscle energy metabolism shown by 31P-MRS in familial hemiplegic migraine. J Neurol Sci 1995; 129: 214-22.

55. Chaban Y, Boekema EJ, Dudkina NV. Structures of mitochondrial oxidative phosphorylation supercomplexes and mechanisms for their stabilisation. Biochim Biophys Acta 2014; 1837: 418-26.

56. Shadel GS, Horvath TL. Mitochondrial ROS signaling in organismal homeostasis. Cell 2015; 163: 560-9.

57. Jimenez L, Laporte D, Duvezin-Caubet S, Courtout F, Sagot I. Mitochondrial ATP synthases cluster as discrete domains that reorganize with the cellular demand for oxidative phosphorylation. J Cell Sci 2014; 127: 719-26.

58. Geyik S, Altunisik E, Neyal AM, Taysi S. Oxidative stress and DNA damage in patients with migraine. J Headache Pain 2016; 17: 10.

59. Alp R, Selek S, Alp SI, Taskin A, Kocyigit A. Oxidative and antioxidative balance in patients of migraine. Eur Rev Med Pharmacol Sci 2010; 14: 877-82.

60. Bauer NC, Corbett AH, Doetsch PW. The current state of eukaryotic DNA base damage and repair. Nucleic Acids Res 2015; 43: 10083-101.

61. Lindahl T. The intrinsic fragility of DNA (Nobel Lecture). Angew Chem Int Ed Engl 2016; 55: 8528-34.

62. Anamika, Khanna A, Acharjee P, Acharjee A, Trigun SK. Mitochondrial SIRT3 and neurodegenerative brain disorders. J Chem Neuroanat 2019; 95: 43-53.

63. Kawamata H, Manfredi G. Proteinopathies and OXPHOS dysfunction in neurodegenerative diseases. J Cell Biol 2017; 216: 3917-29.

64. Lin H, Magrane J, Rattelle A, et al. Early cerebellar deficits in mitochondrial biogenesis and respiratory chain complexes in the KIKO mouse model of Friedreich ataxia. Dis Model Mech 2017; 10: 1343-52.

65. Baldelli S, Aquilano K, Ciriolo MR. PGC-1 $\alpha$ buffers ROS-mediated removal of mitochondria during myogenesis. Cell Death Dis 2014; 5: e1515.

66. Gleyzer N, Scarpulla RC. Activation of a PGC-1-related coactivator (PRC)-dependent inflammatory stress program linked to apoptosis and premature senescence. J Biol Chem 2013; 288: 8004-15.

67. Dong X, Guan X, Chen K, et al. Abnormal mitochondrial dynamics and impaired mitochondrial biogenesis in trigeminal ganglion neurons in a rat model of migraine. Neurosci Lett 2017; 636: 127-33.

68. Gasparini CF, Sutherland HG, Griffiths LR. Studies on the pathophysiology and genetic basis of migraine. Curr Genomics 2013; 14: 300-15.

69. Finnila S, Autere J, Lehtovirta M, et al. Increased risk of sensorineural hearing loss and migraine in patients with a rare mitochondrial DNA variant 4336A $>G$ in tRNAGIn. J Med Genet 2001; 38: 400-5. 
70. Wang Q, Ito $\mathrm{M}$, Adams K, et al. Mitochondrial DNA control region sequence variation in migraine headache and cyclic vomiting syndrome. Am J Med Genet A 2004; 131: 50-8.

71. Zaki EA, Freilinger T, Klopstock T, et al. Two common mitochondrial DNA polymorphisms are highly associated with migraine headache and cyclic vomiting syndrome. Cephalalgia 2009; 29: 719-28.

72. Guo S, Esserlind AL, Andersson Z, et al. Prevalence of migraine in persons with the $3243 \mathrm{~A}>\mathrm{G}$ mutation in mitochondrial DNA. Eur J Neurol 2016; 23: 175-81.

73. Takeshima T, Fukuhara Y, Adachi Y, et al. Leukocyte mitochondrial DNA A to G polymorphism at 11084 is not a risk factor for Japanese migraineurs. Cephalalgia 2001; 21: 987-9.

74. Russell MB, Diamant M, Norby S. Genetic heterogeneity of migraine with and without aura in Danes cannot be explained by mutation in mtDNA nucleotide pair 11084. Acta Neurol Scand 1997; 96: 171-3.

75. Shimomura TKA, Marukawa H, Takahashi K. Mutation in platelet mitochondrial gene in patients with migraine. Cephalalgia 1995; 15: 10.

76. Sparaco M, Feleppa M, Lipton RB, Rapoport AM, Bigal ME. Mitochondrial dysfunction and migraine: evidence and hypotheses. Cephalalgia 2006; 26: 361-72.

77. Finnila S, Hassinen IE, Majamaa K. Phylogenetic analysis of mitochondrial DNA in patients with an occipital stroke. Evaluation of mutations by using sequence data on the entire coding region. Mutat Res 2001; 458: 31-9.

78. Marcuello A, Martinez-Redondo D, Dahmani Y. Human mitochondrial variants influence on oxygen consumption. Mitochondrion 2009; 9: 27-30.

79. Kumar S. Emerging options in multiple myeloma: targeted, immune, and epigenetic therapies. Hematology Am Soc Hematol Educ Program 2017; 2017: 518-24.

80. Irwin MH, Moos WH, Faller DV, Steliou K, Pinkert CA. Epigenetic treatment of neurodegenerative disorders: Alzheimer and Parkinson diseases. Drug Dev Res 2016; 77: 109-23.

81. Karimi N, Tavakoli M, Charati JY, Shamsizade M. Single-dose intravenous sodium valproate (Depakine) versus dexamethasone for the treatment of acute migraine headache: a double-blind randomized clinical trial. Clin Exp Emerg Med 2017; 4: 138-45.

82. Liu F, Ma T, Che X, Wang Q, Yu S. The efficacy of venlafaxine, flunarizine, and valproic acid in the prophylaxis of vestibular migraine. Front Neurol 2017; 8: 524.

83. Yi L, Wu Q, Chen N, et al. Valproate plays a protective role against migraine by inhibiting protein kinase $C$ signalling in nitroglycerin-treated mice. Basic Clin Pharmacol Toxicol 2018; 122: 310-6.

84. Kunder SK, Bairy LK, Arivazhahan A. Effect of sodium valproate and docosahexaenoic acid on pain in rats. J Clin Diagn Res 2017; 11: FF05-8.

85. Zhu MM, Li HL, Shi LH, Chen XP, Luo J, Zhang ZL. The pharmacogenomics of valproic acid. J Hum Genet 2017; 62: 1009-14.

86. Passaro D, Rana G, Piscopo M, Viggiano E, De Luca B, Fucci L. Epigenetic chromatin modifications in the cortical spreading depression. Brain Res 2010; 1329: 1-9.

87. Guo JU, Ma DK, Mo H, et al. Neuronal activity modifies the DNA methylation landscape in the adult brain. Nat Neurosci 2011; 14: 1345-51.

88. Rana G, Donizetti A, Virelli G, et al. Cortical spreading depression differentially affects lysine methylation of $\mathrm{H} 3$ histone at neuroprotective genes and retrotransposon sequences. Brain Res 2012; 1467: 113-9.
89. Gerring ZF, McRae AF, Montgomery GW, Nyholt DR. Genome-wide DNA methylation profiling in whole blood reveals epigenetic signatures associated with migraine. BMC Genomics 2018; 19: 69.

90. van der Wijst MG, Rots MG. Mitochondrial epigenetics: an overlooked layer of regulation? Trends Genet 2015; 31: 353-6.

91. Okano M, Bell DW, Haber DA, Li E. DNA methyltransferases Dnmt3a and Dnmt3b are essential for de novo methylation and mammalian development. Cell 1999; 99: 247-57.

92. Tollefsbol TO, Hutchison CA 3rd. Analysis in Escherichia coli of the effects of in vivo CpG methylation catalyzed by the cloned murine maintenance methyltransferase. Biochem Biophys Res Commun 1998; 245: 670-8.

93. Carelli V, Chan DC. Mitochondrial DNA: impacting central and peripheral nervous systems. Neuron 2014; 84 : 1126-42.

94. Shock LS, Thakkar PV, Peterson EJ, Moran RG, Taylor SM. DNA methyltransferase 1 , cytosine methylation, and cytosine hydroxymethylation in mammalian mitochondria. Proc Natl Acad Sci USA 2011; 108: 3630-5.

95. Ooi SK, Qiu C, Bernstein E, et al. DNMT3L connects unmethylated lysine 4 of histone $\mathrm{H} 3$ to de novo methylation of DNA. Nature 2007; 448: 714-7.

96. Wong M, Gertz B, Chestnut BA, Martin LJ. Mitochondrial DNMT3A and DNA methylation in skeletal muscle and CNS of transgenic mouse models of ALS. Front Cell Neurosci 2013; 7: 279.

97. Shaughnessy DT, McAllister K, Worth L, et al. Mitochondria, energetics, epigenetics, and cellular responses to stress. Environ Health Perspect 2014; 122: 1271-8.

98. Dzitoyeva S, Chen H, Manev H. Effect of aging on 5-hydroxymethylcytosine in brain mitochondria. Neurobiol Aging 2012; 33: 2881-91.

99. Pirola CJ, Scian R, Gianotti TF, et al. Epigenetic modifications in the biology of nonalcoholic fatty liver disease: the role of DNA hydroxymethylation and TET proteins. Medicine (Baltimore) 2015; 94: e1480.

100. Salminen A, Kaarniranta K, Hiltunen M, Kauppinen A. Krebs cycle dysfunction shapes epigenetic landscape of chromatin: novel insights into mitochondrial regulation of aging process. Cell Signal 2014; 26: 1598-603.

101. Veeranki S, Winchester LJ, Tyagi SC. Hyperhomocysteinemia associated skeletal muscle weakness involves mitochondrial dysfunction and epigenetic modifications. Biochim Biophys Acta 2015; 1852: 732-41.

102. Wong VS, Langley B. Epigenetic changes following traumatic brain injury and their implications for outcome, recovery and therapy. Neurosci Lett 2016; 625: 26-33.

103. Mechta M, Ingerslev LR, Fabre O, Picard M, Barres R. Evidence suggesting absence of mitochondrial DNA methylation. Front Genet 2017; 8: 166.

104. Nie L, Wu HJ, Hsu JM, et al. Long non-coding RNAs: versatile master regulators of gene expression and crucial players in cancer. Am J Transl Res 2012; 4: 127-50.

105. Pillar N, Bairey O, Goldschmidt N, et al. MicroRNAs as predictors for CNS relapse of systemic diffuse large B-cell lymphoma. Oncotarget 2017; 8: 86020-30.

106. Leggio L, Vivarelli S, L'Episcopo F, et al. microRNAs in Parkinson's disease: from pathogenesis to novel diagnostic and therapeutic approaches. Int J Mol Sci 2017; 18: E2698.

107. Bandiera S, Ruberg S, Girard M, et al. Nuclear outsourcing of RNA interference components to human mitochondria. PLoS One 2011; 6: e20746. 
108. Plath K, Fang J, Mlynarczyk-Evans SK, et al. Role of histone $\mathrm{H} 3$ lysine 27 methylation in $\mathrm{X}$ inactivation. Science 2003; 300: 131-5.

109. Feng W, Li L, Xu X, Jiao Y, Du W. Up-regulation of the long non-coding RNA RMRP contributes to glioma progression and promotes glioma cell proliferation and invasion. Arch Med Sci 2017; 13: 1315-21.

110. Qian C, Guan M, Si C, Shen H, Jin T, Zhang T. Identification of differentially expressed profiles of IncRNAs and mRNAs in ER-negative and HER-2 positive breast cancer. Arch Med Sci Civil Dis 2017; 2: e148-60.

111. Mercer TR, Neph S, Dinger ME, et al. The human mitochondrial transcriptome. Cell 2011; 146: 645-58.

112. Dong Y, Yoshitomi T, Hu JF, Cui J. Long noncoding RNAs coordinate functions between mitochondria and the nucleus. Epigenetics Chromatin 2017; 10: 41.

113. Yoon JH, Abdelmohsen K, Gorospe M. Functional interactions among microRNAs and long noncoding RNAs. Semin Cell Dev Biol 2014; 34: 9-14.

114. Bayoumi AS, Sayed A, Broskova Z, et al. Crosstalk between long noncoding RNAs and microRNAs in health and disease. Int J Mol Sci 2016; 17: 356.

115. Qiu JJ, Liu YN, Ren ZR, Yan JB. Dysfunctions of mitochondria in close association with strong perturbation of long noncoding RNAs expression in down syndrome. Int J Biochem Cell Biol 2017; 92: 115-20.

116. Matilainen O, Quiros PM, Auwerx J. Mitochondria and epigenetics - crosstalk in homeostasis and stress. Trends Cell Biol 2017; 27: 453-63.

117. Chen SP, Fuh JL, Chung MY, et al. Genome-wide association study identifies novel susceptibility loci for migraine in Han Chinese resided in Taiwan. Cephalalgia 2018; 38: 466-75.

118. Winsvold BS, Bettella F, Witoelar A, et al. Shared genetic risk between migraine and coronary artery disease: a genome-wide analysis of common variants. PLoS One 2017; 12: e0185663.

119. Eising E, Datson NA, van den Maagdenberg AM, Ferrari MD. Epigenetic mechanisms in migraine: a promising avenue? BMC Med 2013; 11: 26.

120. Dykes IM, Emanueli C. Transcriptional and post-transcriptional gene regulation by long non-coding RNA. Genomics Proteomics Bioinformatics 2017; 15: 177-86.

121. Xiong X, Yi C, Peng J. Epitranscriptomics: toward a better understanding of RNA modifications. Genomics Proteomics Bioinformatics 2017; 15: 147-53.

122. Ashrafi G, Schwarz TL. The pathways of mitophagy for quality control and clearance of mitochondria. Cell Death Differ 2013; 20: 31-42.

123. Iacobazzi V, Castegna A, Infantino V, Andria G. Mitochondrial DNA methylation as a next-generation biomarker and diagnostic tool. Mol Genet Metab 2013; 110: 25-34. 